\title{
Squeezed polaritons in confined systems
}

\author{
H. HRADIL, A. QUATTROPANI, V. SAVONA and P. SCHWENDIMANN* \\ Institut de Physique Théorique, EPFL, 1015 Lausanne, Switzerland \\ * GRD/DSA, Papiermühlestrasse 25, 3006 Bern, Switzerland
}

We discuss the statistical properties of polaritons in quantum wells embedded in a microcavity. In particular we evaluate the amount of squeezing of confined exciton polaritons in a GaAs quantum well. The limit of a large cavity is also considered.

Bulk polaritons exhibit non classical statistical properties as squeezing [1], which for strong coupling materials like alcali halides can be relevant. Polaritons in confined systems like quantum wells or quantum wires have already been considered in the literature [2], [3]. In this note we investigate how the dimensions of the material influence the statistical properties of the polariton and in particular of its photon component. We consider the interaction of photons and excitons in the confined system within the Hamiltonian already used by Jorda et al. [4], which describes the interaction of a quantized electromagnetic field with a quasi-two-dimensional polarizable medium. The medium is strongly confined in the growth direction $\mathrm{z}$ and behaves like a two dimensional crystal in the transverse direction. The corresponding Hamiltonian reads,

$H=\sum_{\mathbf{q}} \hbar \omega_{q} A_{\mathrm{q}}^{2^{+}} A_{\mathbf{q}}^{2}+\sum_{\mathbf{Q}, \lambda} \hbar v|\mathbf{Q}| A_{\mathbf{Q}, \lambda}^{1^{+}} A_{\mathbf{Q}, \lambda}^{1}+i \sum_{\mathbf{q}, \mathbf{Q}, \lambda} C_{\mathbf{Q}}^{\lambda}\left(A_{-\mathbf{q}}^{2}-A_{\mathbf{q}}^{2^{+}}\right)\left(A_{\mathbf{Q}, \lambda}^{1}+A_{-\mathbf{Q}, \lambda}^{1^{+}}\right)$,

where $A_{\mathrm{q}^{2+}}$ and $A_{\mathrm{q}}{ }^{2}$ are the creation and annihilation operators of the two-dimensional excitons and $\mathbf{q}$ is the component of the wave vector $\mathbf{Q}$ perpendicular to the $\mathbf{z}$-direction, $\mathbf{k}_{\mathbf{z}}$ is the component of $Q$ in the $z$-direction. $A_{q, \lambda} \lambda^{1+}$ and $A_{q, \lambda} \lambda^{1}$ are the field operators. The coupling constants are defined as

$C_{\mathrm{Q}}^{\lambda}=C_{\mathrm{q}, k_{s}}^{\lambda}=C_{-\mathbf{q},-k_{s}}^{\lambda *}=\sqrt{\frac{4 \pi \hbar \mathrm{v}}{\mathrm{L}^{\prime}|\mathrm{Q}|}} \omega_{q} \mu_{\mathrm{cv}} \cdot \hat{\mathbf{e}}_{\mathrm{q}, k_{s}, \lambda} \frac{1}{C} F(0) \frac{1}{L} \int_{-L / 2}^{L / 2} d z \rho(z) e^{i k_{s} z}$,

where

$\mathrm{v}=\mathrm{c} / \varepsilon_{\infty}$ is the velocity of light in the medium, $\omega_{q}=\omega_{0}+\gamma q^{2}$ is the exciton frequency,

$A_{Q, \lambda}^{1}=A_{q, k_{3}, \lambda}^{1}, A_{-\mathrm{Q}, \lambda}^{1^{+}}=A_{-\mathrm{q}_{1}-k_{z}, \lambda}^{\mathrm{1}^{+}}$and $k_{0}=\omega_{0} / \mathrm{v}$.

This coupling constant is derived from a phenomenological model of the exciton in a quantum 
well as indicated in [2]. The $\mathbf{A}^{2}$ term in the coupling is missing but this does not severely affectthe main results presented here. Considering only one field polarization, we diagonalize the Hamiltonian by using the transformation

$B_{\mathrm{q}}^{l}=\sum_{k_{z}} W_{l}\left(k_{x}, \mathbf{q}\right) A_{\mathbf{q}_{,} k_{z}}^{1}+X_{l}(\mathbf{q}) A_{\mathbf{q}}^{2}+\sum_{k_{z}} Y_{l}\left(k_{x}, \mathbf{q}\right) A_{-\mathbf{q},-k_{s}}^{1^{+}}+Z_{l}(\mathbf{q}) A_{-q}^{2^{+}}$

$l$ indicates the polariton modes and the coefficients are

$Z_{l}(\mathbf{q})=\frac{\omega_{\mathbf{q}}-\dot{\Omega}^{\prime}}{\omega_{q}+\Omega^{l}} X_{l}(\mathbf{q})$,

$W_{l}\left(k_{z}, \mathbf{q}\right)=\frac{-i}{\hbar} \frac{2 \omega_{q}}{\omega_{q}+\Omega^{l}} \frac{C_{\mathbf{q}, k_{z}}^{\lambda}}{\mathrm{v}|\mathbf{Q}|-\Omega^{l}} X_{l}(\mathbf{q})$,

$Y_{l}\left(k_{z}, \mathbf{q}\right)=\frac{\mathbf{v}|\mathbf{Q}|-\Omega^{l}}{\mathrm{v}|\mathbf{Q}|+\Omega^{l}} W_{l}\left(k_{z}, \mathbf{q}\right)$,

where $X(q)$ is determined from $\left[B_{q}^{l}, B_{q}^{l^{\prime+}}\right]=\delta_{l, l^{\prime}}$.

The quantity $\Omega^{l}$ is the polariton energy and is evaluated from the dispersion formula

$\Omega^{2}-\omega_{q}^{2}+\frac{4 \omega_{q}}{\hbar^{2}} \sum_{k_{s}} \frac{\mathbf{v}|\mathbf{Q}|}{\mathrm{v}^{2}|\mathbf{Q}|^{2}-\Omega^{2}}\left|C_{\mathbf{q}, k_{s}}^{\lambda}\right|^{2}=0$

$|\mathbf{Q}|=\sqrt{k_{z}^{2}+q^{2}}$.

At this point it is important to distinguish between a finite cavity for which $k_{z}$ takes discrete values and the continuum case.

For $\mathrm{k}_{\mathrm{z}}=\pi(2 \mathrm{n}+1) / \mathrm{L}^{\prime}$ the polariton energy is always different from the photon energy and the denominator in (5) never vanishes. Therefore, small cavity strongly differs from the continuum case. From (7) we obtain a lower polariton branch $\Omega^{(L P)}(q)$ with $q>\Omega / v$ and a series of upper polaritons, one branch $\Omega l(\mathrm{UP})(\mathrm{q})$ for each value of $l$ and with $\mathrm{q}<\Omega / \mathrm{v}$.

The dispersion of the upper polaritons in the finite cavity case show a peculiar behaviour. We consider a cavity of length $L^{\prime} \approx 2 \pi / k_{0} \gg L$. The dispersion (7) is expressed in analytical form as

$\omega^{2}-1=8 \pi \frac{v}{c^{2}} \frac{\left|\mu_{\mathrm{cv}}\right|^{2}|F(0)|^{2}}{\hbar \alpha^{3} L^{2} k_{0}^{2}}\left[\alpha L k_{0}-\tan \left(\alpha L^{\prime} k_{0} / 2\right)\left(1-\cos \left(\alpha L k_{0}\right)\right)-\sin \left(\alpha L k_{0}\right)\right]$.

In (9) normalized quantities are used, i.e. $\omega=\Omega / \omega_{0}$ and $\alpha=\sqrt{\left|\omega^{2}-q^{2} / k_{0}^{2}\right|}$.

The solutions lead to the dispersion curves shown in Fig. 1; these are calculated for a GaAs quantum well of width $L=60 \AA$ and for a cavity of length $L^{\prime}=100 \mathrm{~L}$ with perfectly reflecting walls and a vanishing electric field on the walls. Cavity and QW have the same dielectric constant $\varepsilon=5$ and an exciton energy of $1.6 \mathrm{eV}$ is chosen. We use the relation

$\left|\boldsymbol{\mu}_{\mathrm{cv}}\right|^{2}|F(0)|^{2} / \hbar=f e^{2} / 2 m \omega_{0}$

between the exciton oscillator strength $f$ and the quantities used here, and take from Tassone et al. [3] $f=3610^{-5} \AA^{-2}$. The details of the dispersion are presented in Fig. 2 for the second branch showing an anticrossing behaviour. This result is reminescent of the so called vacuum Rabi 


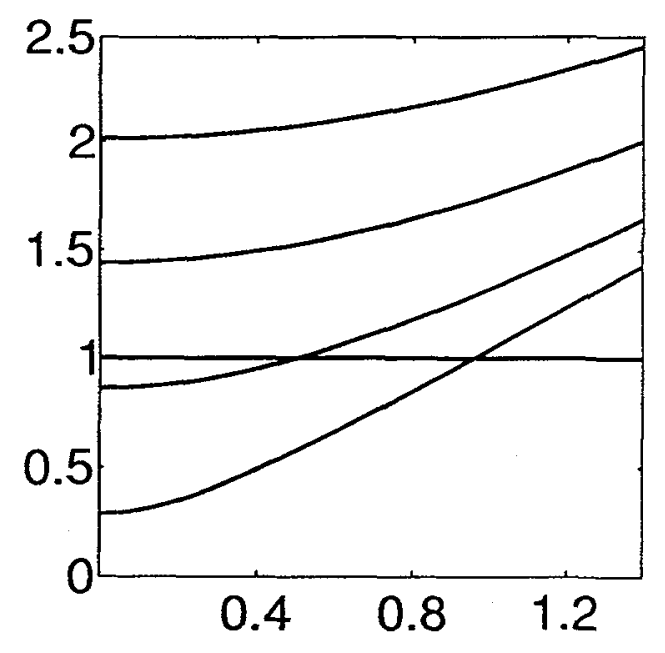

Fig. 1. Dispersion curves for the upper polariton branches. The normalized frequency $\Omega l$ (UP) $/ \omega_{0}$ as a function of $q / \mathrm{k}_{0}$ is evalueted for a $Q W$ of $60 \AA$. Parameters appropriate to GaAs are used.

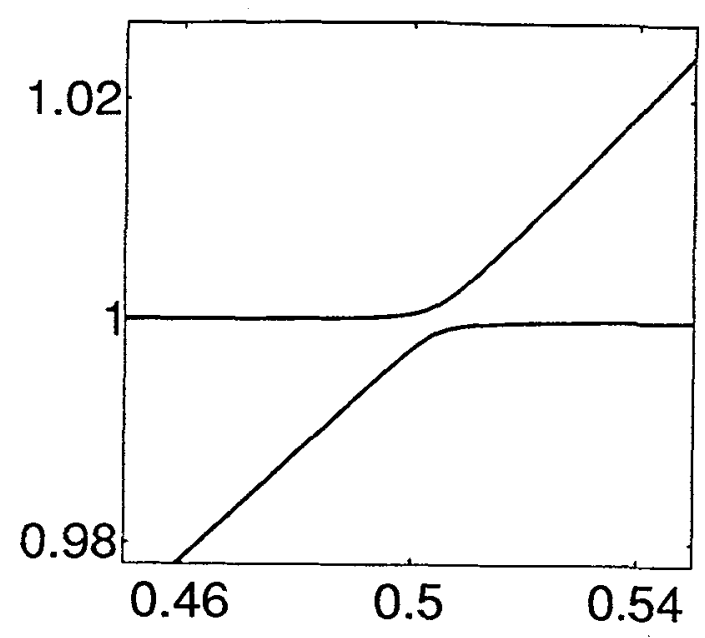

Fig. 2. A detail of the dispersion curves relative to the anticrossing point of the second branch.

splitting known from cavity quantum electrodynamics. For larger values of the cavity length $\mathrm{L}^{\prime}$ the number of anticrossing points grows as the spacing between the photon energies becomes smaller. It is also found that the maxium of the density of states $\mathcal{D}(\Omega)$ for a given upper polariton branch lies on the classical resonance curve [2] and that this density of states has the same width as the classical resonance. For the continuum case we obtain again a lower polariton branch, which does not differ notably from the discrete case. For the upper polariton the situation is totally different, because when the discrete sum goes over to an integral the integrand becomes singular. In this case $\Omega>v q$ and therefore it exist $\mathrm{k}_{\mathrm{z}}$ such that $\mathrm{v}|\mathrm{Q}|=\Omega>\mathrm{vq}$. When this relation is satisfied the polariton states are degenerate with the photon-continuum giving rise to a Fano resonance. This result has been found, with a different interpretation, quantum mechanically by Jorda et al. [4] and even classically by Tassone et al [3]. The classical resonance of [2] is found in this context to correspond to the value of the polariton energy for which the number of upper polaritons is maximum. Furthermore the Fano width is the same as the radiative damping width for the classical resonance.

We also calculate the squeezing by defining, as usual for two-mode systems, the operator

$$
d_{2}=\frac{-i}{2^{3 / 2}}\left[B_{q}^{1}+B_{-q}^{1}-B_{-q}^{1^{+}}-B_{q}^{1^{+}}\right]
$$

and evaluating the quantity

$$
\begin{aligned}
\left.\left.<\left(\Delta d_{2}\right)^{2}\right\rangle \equiv<0\left|\left(\Delta d_{2}\right)^{2}\right| 0\right\rangle= & \frac{1}{4}\left[\left|Z_{1}(\mathbf{q})\right|^{2}+\left|X_{1}(\mathbf{q})\right|^{2}+\sum_{k_{z}}\left(\left|W_{1}\left(k_{z}, \mathbf{q}\right)\right|^{2}+\left|Y_{1}\left(k_{z}, \mathbf{q}\right)\right|^{2}\right\}\right] \\
& -\frac{1}{2} \Re e X_{1}(\mathbf{q}) Z_{1}(\mathbf{q})-\frac{1}{2} \Re e \sum_{k_{z}} W_{1}\left(k_{z}, \mathbf{q}\right) Y_{1}\left(-k_{z},-\mathbf{q}\right)
\end{aligned}
$$


where $|0\rangle$ is the free vacuum. The square root of this quantity gives the amount of squeezing present in the system. We evaluate the amount of squeezing in both the finite and the infinite cavity configurations. The finite cavity configuration is more suited for the upper polariton squeezing due to the appearence of a discrete set of modes.

Squeezing in the $\mathrm{d}_{2}$ quadrature component i.e.

$$
\Delta_{s q} \equiv\left[1-\sqrt{\frac{\left.<\left(\Delta d_{2}\right)^{2}\right\rangle}{1 / 4}}\right] \times 100
$$

is presented in Fig. 3 for the first polariton branch as a function of the wave vector q. Its magnitude of about $0.09 \%$ is small but larger than in the bulk case as a consequence of a larger value of the oscillator strength of the confined exciton. The amount of squeezing has been evaluated in the continuum case and has a value comparable to that of the discrete upper polariton.

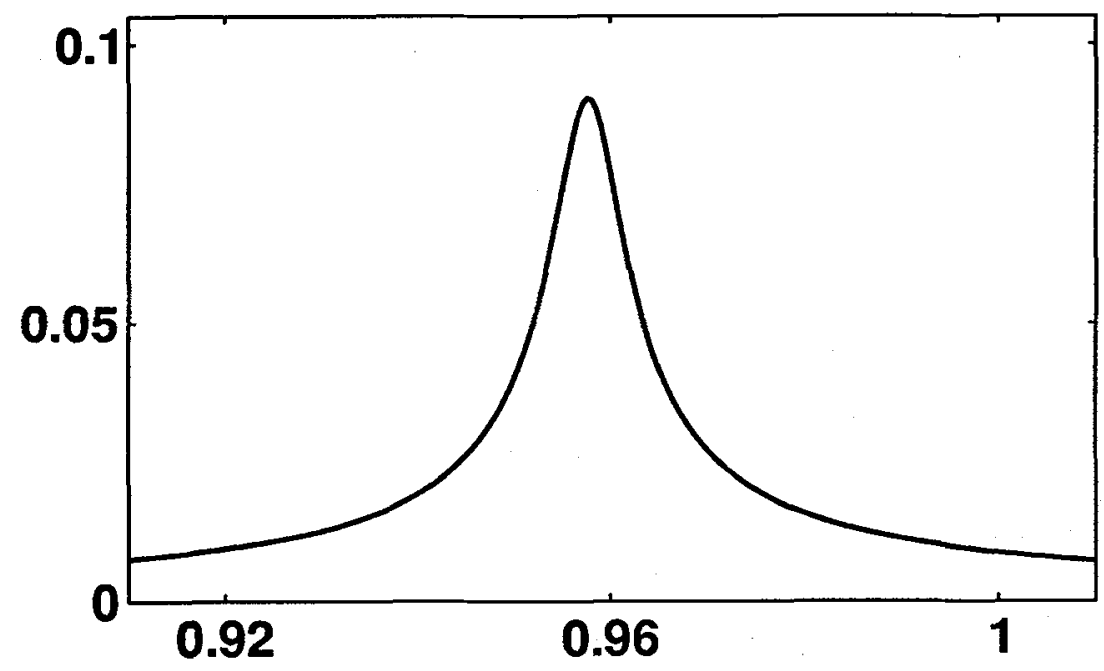

Fig. 3. Squeezing percentage $\Delta_{s q}$ of the $d_{2}$ operator for the first polariton branch as a function of the normalized wave vector $q / k_{0}$.

References:

[1] Schwendimann P. and Quattropani A., Europhys. Letters 17 (1992) 355-359.

[2] Tassone F., Bassani F. and Andreani L. C., Il Nuovo Cimento D 12 (1990) 1673-1687.

[3] Tassone F. and Bassani F., Il Nuovo Cimento D 14 (1992) 1241-1256.

[4] Jorda S., Rössler U. and Broido D., Superlattices and Microstructures 12 (1992) 85-87. 